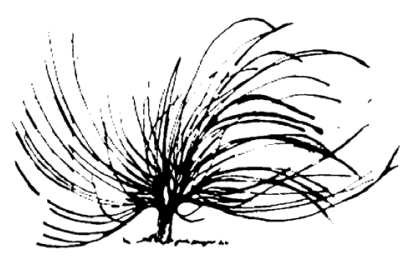

\title{
Caracterización de la evaluación de los aprendizajes en una universidad con modalidad a distancia: el caso de la Universidad Estatal a Distancia (UNED)

\author{
Yency Calderón Badilla ${ }^{1}$ \\ Universidad Estatal a Distancia \\ Escuela de Ciencias Exactas y Naturales \\ Costa Rica \\ ycalderon@uned.ac.cr \\ Rosita Ulate Sánchez ${ }^{2}$ \\ Universidad Estatal a Distancia \\ Escuela de Ciencias Exactas y Naturales \\ Costa Rica \\ rulate@uned.ac.cr
}

\begin{abstract}
Resumen
La evaluación de los aprendizajes se desarrolla en función de los lineamientos y la normativa institucional de una entidad de educación y son el reflejo de una estrategia pedagógica y didáctica. Por tal motivo, se realizó, en el 2017, una investigación cualitativa con un análisis comparativo de varios documentos institucionales de la Universidad Estatal a Distancia de Costa Rica. Se plantearon preguntas guiadoras en relación con la evaluación de los aprendizajes: ¿Cómo debe ser la evaluación de los
\end{abstract}

\section{(ब) $(\Theta \Theta$}

Recibido: 9 de mayo de 2019. Aprobado: 29 de abril de 2020.

http://dx.doi.org/10.15359/rep.15-2.8

1 Profesora, Máster en Administración Educativa, Máster en Administración Universitaria, Máster en Evaluación Educativa de la Universidad de Costa Rica.

2 Profesora. Doctora en Educación de Nova Southeastern University y Maestría en Administración de la Universidad de Costa Rica. 
aprendizajes?, ¿al servicio de quién está?, ¿cuál es su planificación y función?, y ¿qué tipo de evaluación se da? Aunque se encontraron coincidencias en las estrategias de auto y coevaluación, no existe un único concepto institucional en cuanto a la evaluación de los aprendizajes, pero sí está al servicio del estudiante con un propósito orientador, preventivo y de realimentación correctiva. Sin embargo, se requiere concordancia de las características y atributos que se expresan en la normativa con la planificación de la evaluación, especialmente en los mecanismos de control del cumplimiento esperado. En un modelo de educación a distancia, el enfoque de evaluar para aprender pareciera ser más pertinente, dado que incluye la evaluación diagnóstica, formativa, formadora y sumativa mediante estrategias alternativas que posibilitan la autorregulación del estudiante.

Palabras clave: educación a distancia, evaluación de los aprendizajes, lineamientos, normativa, TIC

\begin{abstract}
The evaluation of learning is developed according to the guidelines and institutional regulations of an educational entity and is a reflection of a pedagogical and didactic strategy. For this reason, in 2017, a qualitative research was carried out with a comparative analysis of several institutional documents of Universidad Estatal a Distancia of Costa Rica. Guiding questions were stated in relation to the evaluation of learning: How should the evaluation of learning be?, who does it serve?, what is its planning and function?, and what type of evaluation is given? Although coincidences were found in the self-assessment and peer assessment strategies, there is no single institutional concept regarding the evaluation of learning, but it is at the service of the students with a guiding, preventive, and corrective feedback purpose. However, agreement is required on the characteristics and attributes that are expressed in the regulations with the planning of the evaluation, especially in the mechanisms of control for expected compliance. In a distance education model, the approach of evaluating to learn seems to be more relevant, given that it includes diagnostic, formative, self-regulated learning, and summative evaluation
\end{abstract}


through alternative strategies that enable self-regulation of the students.

Keywords: evaluation of learning, distance education, ICT, guidelines, regulations.

\section{Introducción}

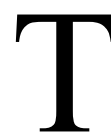

Tanto Barberà, Romiszouki, Sangrà y Simonson (2014), como Juca (2016) coinciden en que la educación a distancia (EaD) reúne varias características que la definen: es un método educativo formal de estudio independiente, avalado por una institución educativa, en la cual los sujetos de aprendizaje están separados de los recursos y estos, a su vez, son mediados de forma planificada a través de los sistemas de comunicación e información disponibles.

La educación a distancia cambió los esquemas tradicionales en los procesos de enseñanza y aprendizaje. Por su parte, los avances en las tecnologías de información y comunicación (TIC) han venido a potencializar sus dimensiones de flexibilidad en la mediación didáctica. Por estas razones, tanto Juca (2016) como Quesada (2006) afirman que en la educación a distancia, el uso de las TIC se vuelve esencial durante el proceso de enseñanza y aprendizaje del estudiante, en un programa de estudio, así como en la actualización y en su desarrollo profesional continuo.

Para Quesada (2006), en la educación a distancia la mediación se da en todos los recursos disponibles para el estudiante y el docente. Además, es un pilar fundamental en la evaluación de los aprendizajes para analizar y valorar "las ejecuciones para consolidar o reorientar los logros" (p. 3). De tal manera, en el contexto de la educación a distancia, la modalidad de evaluación de los aprendizajes puede ser realizada de manera tradicional, es decir, presencialmente, o mediante estrategias alternativas que permiten las TIC al integrar el uso de evaluaciones en línea o virtuales. En este sentido, algunas de las innovaciones en la tecnología educativa han permitido desarrollar actividades evaluativas, tales como el uso de pruebas en línea con ítems cerrados y abiertos, wikis, blogs, videos, audios, chats, foros y la interacción (profesor-alumno) en tiempo real, entre otros.

La evaluación de los aprendizajes es un tema de discusión necesaria en la educación superior, ya que esta es responsable de certificar la credibilidad de las personas graduadas, lo cual se lleva a cabo mediante la formación profesional; que conlleva una rigurosa valoración 
del logro de aprendizaje y el alcance que se tenga de los perfiles de egreso. Por lo tanto, este artículo como parte de una investigación realizada en el año 2017 tiene como propósito desarrollar algunos aspectos conceptuales que deben estar presentes en la normativa institucional relacionada con la evaluación de los aprendizajes, como lo son: ¿Al servicio de quién está?, ¿cómo es la planificación?, ¿cuál es la función? $\mathrm{y}$ ¿quiénes son los agentes de la evaluación?

\section{Marco contextual}

En Costa Rica, la educación superior universitaria con modalidad a distancia surgió en 1977 con la creación de la Universidad Estatal a Distancia, mediante la Ley 6044, aprobada por la Asamblea Legislativa y publicada en La Gaceta N. ${ }^{\circ} 50$ del 12 de marzo de 1977. Es así que la UNED se fundó como una alternativa de expansión educativa formal y no formal mediante el uso de técnicas de comunicación social. Desde entonces, la UNED ha establecido estrategias y normativa que regulan su quehacer académico y en especial, el proceso de evaluación de los aprendizajes.

La UNED posee diferentes documentos relacionados con la evaluación de los aprendizajes, por lo que fue importante analizarlos en función de la coherencia de la normativa institucional, en relación con las nuevas tendencias en evaluación y los entornos virtuales en los modelos de educación superior a distancia. Dicho análisis permitió señalar las acciones de mejora en el quehacer de las diferentes dependencias académicas que integran la labor en este proceso, y, por ende, en las prácticas evaluativas que se diseñan e implementan en las diferentes asignaturas de los programas de estudio, desde un enfoque más flexible, crítico, técnico y ético.

\section{Marco teórico}

La evaluación de los aprendizajes en cualquier sistema educativo posee diversidad de aristas relacionadas con los actores que participan en el proceso didáctico y este es un tema relevante. Por ejemplo, para Perazzi y Celma (2017), de la evaluación de los aprendizajes "dependen la certificación y acreditación necesarias de los aprendizajes para la formación de un profesional egresado universitario" (p. 24), mientras que para Flores (2010) la importancia radica en su potencial de reflexión para "emitir juicios de valor y tomar decisiones pertinentes y oportunas para optimizar el aprendizaje, (...) toma de decisiones propia 
de la evaluación debe basarse en criterios previamente establecidos, los cuales se obtienen a partir de los objetivos de aprendizaje" (p. 2).

Acerca de la evaluación de los aprendizajes en educación a distancia con la incorporación de las TIC, se ha potenciado la comunicación sincrónica y asincrónica con el estudiantado, lo que permite implementar estrategias alternativas de evaluación, como la evaluación auténtica y la evaluación integral, entre otras, que implican la participación del grupo docente y estudiantil. De acuerdo con Quesada (2006), en la educación a distancia mediada por entornos virtuales, se hace necesaria la evaluación continua del aprendizaje y la implementación de diferentes instrumentos que permitan visualizar el grado de avance y el logro de cada estudiante en los diversos niveles.

Morgan y O'Reilly (2002) establecen seis aspectos claves que deben cumplirse en la evaluación de los aprendizajes en la educación a distancia:

1. Las tareas evaluativas orientan el aprendizaje, por lo tanto, deben ser significativas y desarrollar las habilidades deseadas.

2. El propósito, los valores, los criterios y los estándares utilizados para juzgar el logro deben ser explícitos, tanto para el docente como para el estudiante.

3. El uso de evaluación autentica y holística contribuye en la motivación y la formación del estudiante; las evaluaciones aplicadas como estudios de caso, escenarios y proyectos crean escenarios reales y cercanos a su contexto.

4. Se deben planificar acciones y actividades de autodirección, pensamiento crítico, autogestión, y autoevaluación para que el estudiante asuma la responsabilidad y el control de su aprendizaje.

5. Se debe implementar suficiente evaluación formativa y a tiempo.

6. La planificación de la evaluación de los aprendizajes debe realizarse considerando el contexto de los estudiantes, así como su percepción acerca de las tareas de evaluación. (p. 15)

Es importante destacar que, ante las nuevas tendencias conceptuales de la evaluación de los aprendizajes, se están gestando nuevas prácticas evaluativas que se pueden vincular al proceso de formación integral de estudiantes. Por ejemplo, la evaluación diagnóstica, formativa, formadora, autoevaluación, coevaluación y evaluación continua. 
La evaluación diagnóstica se realiza al inicio de un periodo académico o de una asignatura, su objetivo es obtener información de cada estudiante referida a conocimientos previos y aspectos personales o de contexto, la cual permita orientar la planificación didáctica en las asignaturas, los cursos o las unidades de aprendizaje. Lo importante, tal y como lo expone Esquivel (2009), es que una vez emitido un juicio de valor mediante la aplicación de un instrumento (por lo general un examen), se tomen decisiones pedagógicas. De tal manera que esos conocimientos previos con que cuenta el estudiante sirvan como fuente para la toma de decisiones en el desarrollo de un plan didáctico, sea de un plan de clase, una unidad, un material didáctico o una asignatura. Sin embargo, Pimienta (2008) plantea que la evaluación diagnóstica "no solo debe consistir en aplicar un examen, pues, además existen variantes muy efectivas para recopilar información que ayudaría a explicar mucho de lo que ha sucedido con el aprendizaje de los estudiantes" (p. 38); por ejemplo, en el nivel universitario en un modelo a distancia puede solicitarse a los estudiantes que realicen un blog personal que incluya una presentación de sus quehaceres cotidianos, lo que le gusta como pasatiempo, el contexto en el cual vive, sus experiencias como estudiante, lo que considera como fortalezas personales, sus limitaciones y retos, es decir información que refleje la parte más humana del estudiante. Solicitar un portafolio al inicio del curso lectivo o a la mitad, también permite tener una idea más clara de los intereses y aprendizajes del estudiantado.

En cuanto a la evaluación formativa, Medina, Cardona, Castillo y Domínguez (1998) afirman que:

Este tipo de evaluación pretende evaluar el proceso que el alumno sigue cuando se aproxima a un concepto, en el dominio de procedimientos o en el descubrimiento y eventual adhesión a unos valores. La evaluación pretende modificar y perfeccionar sobre la marcha todo lo que no se ajusta a los objetivos. (p. 209)

La evaluación formativa tiene el propósito de orientar, a partir de la identificación de las limitaciones o errores, a los estudiantes para que mejoren sus actuaciones académicas en las estrategias e instrumentos de evaluación (Reina, 1993, según se cita en Castillo, 2002). Esta misma autora sostiene que la evaluación formativa pretende detectar "tanto las 
mejoras y los progresos, como las carencias o los inconvenientes" (p. 86), por lo que son los estudiantes los que se benefician de esta evaluación.

Por su parte, la evaluación formadora tiene el propósito de potenciar en el estudiantado acciones metacognitivas en las que sean capaces de autorregular su aprendizaje, reflexionar e identificar sus errores y plantear las alternativas de solución para la mejora continua de su proceso de enseñanza y aprendizaje. Al respecto, Moraza (s. f.), según se cita en López (2009) identifica la evaluación formadora como "una estrategia de evaluación dirigida a promover el autorreflexión y el control sobre el propio aprendizaje. Para ello se pueden aplicar tres técnicas: autoevaluación, evaluación mutua y coevaluación" (p. 34). Por su parte, Sanmartí, Jorba e Ibáñez (2002) afirman que este tipo de evaluación "pretende formar al alumno en sus procesos de pensamiento y de aprendizaje ayudándole a construir su propio sistema personal de aprendizaje" (p. 303).

La evaluación sumativa se refiere básicamente a productos finales que han sido medibles y observables en el estudiantado con el objetivo de acreditar-certificar o calificar su nivel de logro. Esquivel (2009) la denomina como "acumulativa" o de "resultado" y la define como "aquella que se realiza al terminar un periodo determinado de los procesos de enseñanza y aprendizaje. Tiene como propósito principal calificar el grado del desempeño de los estudiantes, el logro de los objetivos curriculares" (p. 24). De Ketele y Roegiers (1995) plantean que la evaluación sumativa es una "modalidad de evaluación asociada a menudo con la certificación, ya que pone el acento en la suma de los logros y conlleva la idea de balance. Es control final que desemboca en la superación o no de una acción" (p. 54). Estos autores consideran que implica momentos de control en el "destino" del estudiantado, en la mayoría de los casos, las acciones de mejora o paliativas, ya no tienen sentido en el momento que se les asigna la calificación final.

La autoevaluación sucede cuando la persona realiza una valoración de sus propias acciones o comportamientos, y progresos. Por lo tanto, el agente de la evaluación y su objeto se identifican (Casanova, 1995). Al trasladar la autoevaluación al campo educativo, docentes y estudiantes se convierten en sus propios agentes evaluadores. Medina, Cardona, Castillo y Domínguez (1998), así como Castillo y Cabrerizo (2007) la plantean como una modalidad motivadora y formativa para el estudiantado, ya que produce estímulo para lograr la superación personal a partir de la identificación de los propios logros y carencias. 
Por otra parte, la coevaluación consiste en la evaluación entre pares o revisión mutua que se da en un espacio colaborativo de aprendizaje de manera sincrónica o asincrónica. También puede presentarse en diversas circunstancias colectivas que el docente haya planificado para cumplir con el logro de ciertos objetivos de aprendizaje. Para Castillo y Cabrerizo (2009), la coevaluación se "refiere a la evaluación grupal y entre pares que realizan los alumnos de su actividad escolar. También del profesor con sus alumnos" (p. 193). Al respecto, es necesario dar a conocer, desde el principio, a los involucrados los criterios de coevaluación. Al respecto, Pimienta (2008) destaca que se debe dejar claro que no se evalúa a la persona, sino solo aquello que se realiza y plantea, en la medida de lo posible. De modo que se resalte, en su mayoría, aquellos aspectos positivos y logros que ha tenido el otro.

Por su parte, la evaluación continua es aquella que se realiza durante todo un proceso educativo que responde a una planificación de la misma. Los autores Rotger (1992) y Cardona (1994) coinciden en que este tipo de evaluación está integrada a lo largo de todo el proceso de enseñanza y aprendizaje, y es intrínseca en toda la dinámica. Mientras que Reina (1993) afirma que la evaluación continua "se produce en el transcurso de un proceso o de una actividad para conocer cómo se está desarrollando en referencia a un plan definido previamente. Supone la constatación permanente de la evolución de los alumnos" (p. 257). Por lo tanto, los resultados permiten observar, reflexionar y tomar decisiones en relación con el nivel de avance del estudiantado, ya sea de manera individual o en grupo.

\section{Metodología}

Este estudio formó parte de los objetivos de la tesis de maestría denominada: Valoración de las implicaciones éticas, sociales y didácticas de la evaluación de los aprendizajes apoyada en entornos virtuales (Calderón, 2017). La investigación se realizó a partir de un enfoque cualitativo con un alcance descriptivo, por medio de un análisis de documentos institucionales como unidades de análisis (Modelo Pedagógico de la UNED, 2004; el Reglamento General Estudiantil, $2012^{\mathrm{a}}$; el Plan de Desarrollo Académico 2012-2017, 2012b y el documento titulado Cómo diseñar y ofertar cursos en línea, 2011. De acuerdo con Cook y Reichardt (2005) este tipo de estudios documentales se utiliza cuando: 
La investigación necesita hacerse evaluativa, donde es necesario sacar a la luz y tener en cuenta la multiplicidad de factores y de valores que entran en conflicto en cualquier realidad social y a la que el propio investigador no es ajeno. (p. 18)

Para efectos de este estudio, se definió que la caracterización de la evaluación de los aprendizajes se refiere a las atribuciones o las características que la normativa institucional de la UNED le otorga a dicha evaluación. Para obtener los datos, se ejecutaron la revisión y el análisis de la normativa institucional referida a la evaluación de los aprendizajes, al seguir una plantilla para sistematizar las principales características, por lo que se establecieron las siguientes categorías de análisis, a manera de pregunta, las cuales permitieron organizar la información: a) ¿Cómo debe ser la evaluación de los aprendizajes?, b) ¿al servicio de quién está la evaluación de los aprendizajes?, c) ¿cuál es la planificación de la evaluación de los aprendizajes?, d) ¿cuál es la función de la evaluación?, y e) ¿cuál es el tipo de evaluación según sus agentes?

\section{Resultados y discusión}

\section{Modelo Pedagógico de la UNED}

E1 Modelo Pedagógico (UNED, 2004) establece los principios y los criterios que orientan cada una de las actividades de enseñanza y de aprendizaje en la UNED. Tiene como propósito responder a lineamientos generales enfocados en ser una guía para presidir dichos procesos. También se declara ser vigilante de la calidad y la excelencia académica de los programas y, a la vez, ser el modelo que orienta con criterio pedagógico el amplio uso de medios y apoyos didácticos. Otra función de dicho documento consiste en trascender de lo didáctico hacia la formación integral y permanente, y el pleno desarrollo de potencialidades desde la ética personal y el compromiso social (UNED, 2004).

En el caso de la evaluación de los aprendizajes, el Modelo Pedagógico se adjudica el hecho de promover la autorregulación desde las prácticas evaluativas desarrolladas en las carreras. Además, invita a que el grupo docente sea un medio para regular el proceso de aprendizaje mediante la retroalimentación y la orientación académica adecuadas.

El Modelo Pedagógico de la UNED (2004) hace un llamado a considerar que la evaluación de los aprendizajes, más que un momento 
de medición, es una estrategia didáctica que debe generar en el estudiantado la autonomía y la capacidad de autorregular su aprendizaje. Además, la concibe como un elemento orientador, que es guía en el diseño de materiales, en el planeamiento de las asignaturas y en la acción de mediación, facilitación y apoyo al proceso de aprendizaje. Cabe destacar que en el apartado de evaluación de los aprendizajes no se hace referencia a la evaluación apoyada en entornos virtuales ni a otros medios tecnológicos para operacionalizarla. Se destaca que la evaluación en el Modelo pedagógico es un elemento que orienta y da apoyo al proceso de aprendizaje. Igualmente, consiste en una estrategia didáctica $\mathrm{y}$ tiene una función autorreguladora en el estudiante.

En la Tabla 1, se observan las principales características que el Modelo Pedagógico (UNED, 2004) le atribuye a la evaluación de los aprendizajes. Tales características son clasificadas a partir de algunas consideraciones relacionadas con su función. Por ejemplo, ¿al servicio de quién está? Aspectos que de acuerdo con Flores (2010) brindan lineamientos oportunos para la toma de decisiones en la evaluación de los aprendizajes.

\section{Tabla 1.}

Caracterización de la evaluación de los aprendizajes según el Modelo Pedagógico de la UNED (2004)

\begin{tabular}{|c|c|}
\hline Consideración & Características \\
\hline $\begin{array}{l}\text { ¿Cómo debe ser la } \\
\text { evaluación de los } \\
\text { aprendizajes? }\end{array}$ & $\begin{array}{ll}\text { - } & \text { Integral } \\
\text { - } & \text { Integrada } \\
\text { - } & \text { Formativa } \\
\text { - } & \text { Ruguladora de los aprendizajes. } \\
\text { - No es separada del proceso. } \\
\text { - No es un momento de comprobación. } \\
\text { - Requiere validez. } \\
\text { No se limita a una repetición de los puntos de vista } \\
\text { ofrecidos en los materiales didácticos. } \\
\end{array}$ \\
\hline $\begin{array}{l}\text { ¿Al servicio de quién } \\
\text { está la evaluación de los } \\
\text { aprendizajes? }\end{array}$ & $\begin{array}{l}\text { - Lo lleva a cabo el estudiante. } \\
\text { - Supone la participación del estudiantado para } \\
\text { apropiarse de los objetivos de aprendizaje. } \\
\text { - Procura la formación de un estudiante autónomo y } \\
\text { capaz de seguir aprendiendo solo. }\end{array}$ \\
\hline
\end{tabular}




\begin{tabular}{|c|c|}
\hline Consideración & Características \\
\hline $\begin{array}{l}\text { ¿Cuál es la planificación } \\
\text { de la evaluación de los } \\
\text { aprendizajes? }\end{array}$ & $\begin{array}{l}\text { - Es un elemento que da dirección y sentido al diseño de } \\
\text { materiales, a la programación de cursos, a la acción de } \\
\text { facilitación y al apoyo para el aprendizaje. } \\
\text { - Cuenta con características técnicas de los instrumentos } \\
\text { utilizados, incluye los criterios y la forma de } \\
\text { aplicarlos. } \\
\text { - Los instrumentos de evaluación son elaborados } \\
\text { y calificados por personas diferentes, debido a su } \\
\text { modelo a distancia. }\end{array}$ \\
\hline $\begin{array}{l}\text { ¿Cuál es la función de } \\
\text { la evaluación? }\end{array}$ & $\begin{array}{l}\text { - Reguladora durante el proceso de aprender. } \\
\text { - Promueve la autorregulación. } \\
\text { - } \text { accionta la planificación autónomamente de las } \\
\text { - Es una estrategia didáctica más allá que una medición } \\
\text { o comprobación de resultados. } \\
\end{array}$ \\
\hline $\begin{array}{l}\text { ¿Qué tipo de evaluación } \\
\text { se da según sus } \\
\text { agentes? }\end{array}$ & - Propicia la coevaluación entre pares de estudiantes. \\
\hline
\end{tabular}

Nota: Adaptado del Modelo Pedagógico (UNED, 2004), análisis hecho en 2017.

\section{Reglamento General Estudiantil (UNED)}

Este Reglamento (2012a) norma en su capítulo IX lo relacionado con la evaluación de los aprendizajes del estudiantado. Dicho capítulo establece una conceptualización de términos evaluativos. A su vez, determina el tipo de instrumentos que se pueden utilizar, así como el valor máximo porcentual que se les otorga. También regula aspectos relacionados con la aplicación de pruebas escritas presenciales y con el debido proceso para apelar los resultados de evaluación, según los instrumentos. En la UNED, la evaluación de los aprendizajes demanda un seguimiento individualizado para cada estudiante a través de diversas estrategias e instrumentos evaluativos que permitan obtener información relevante de su proceso de aprendizaje para certificar cuantitativamente su nivel de logro. Igualmente, se delega a la evaluación de los aprendizajes dos funciones clave: por un lado, informar al estudiante el nivel de logro y certificar su aprendizaje; por otro lado, considerar sus resultados para incorporar cambios que promuevan la autorregulación y el aprendizaje significativo, aspectos consecuentes con lo señalado por Morgan y O'Reilly (2002) en el establecimiento de criterios estándares de autogestión para el estudiante. 
Paralelamente, la UNED declara la evaluación de los aprendizajes como de carácter riguroso, sistemático, integrado, formativo y sumativo. En este sentido, se abarcan las condiciones técnicas de los instrumentos de evaluación desde la validez y la confiabilidad. Además, es importante considerar el uso de los resultados, de manera que se aprovechen para regular el proceso de enseñanza y acreditar el logro de la comunidad de estudiantes.

También, en el RGE, se hace mención a los tipos de evaluaciones que deben ser planificadas e informadas a cada estudiante. Sin embargo, no hay mayor detalle para comprender a qué se refiere o qué implica dicha planificación.

El RGE (UNED, 2012a) define la evaluación de los aprendizajes como "un proceso sistemático de obtención de información válida y útil para formular juicios valorativos acerca de los efectos del proceso de formación en cada estudiante" (p. 28). A la vez, el mismo reglamento destaca que la evaluación de los aprendizajes cumple con dos funciones: la primera consiste en informar a los estudiantes sobre su progreso académico, para determinar el cumplimiento de los objetivos y las competencias que deben ser demostradas, de manera que sean acreedores de una certificación de cumplimiento del plan de estudios. La segunda implica determinar las dificultades de cada estudiante en el proceso de aprendizaje, así como sus logros, con el propósito de hacer cambios y mejoras en pro de un aprendizaje significativo, así como del empoderamiento de un aprendizaje autorregulado, propio del modelo de educación a distancia (UNED, 2012a).

En la Tabla 2 se presenta el resumen de la caracterización que le otorga el RGE (UNED 2012a) a la evaluación de los aprendizajes, en ella se da énfasis a los aspectos técnicos y a la planificación que debe hacerse de ella. 
Tabla 2.

Caracterización de la evaluación de los aprendizajes según el RGE (UNED, 2012a)

\begin{tabular}{|c|c|}
\hline Consideración & Característica \\
\hline $\begin{array}{l}\text { ¿Cómo debe ser la } \\
\text { evaluación de los } \\
\text { aprendizajes? }\end{array}$ & 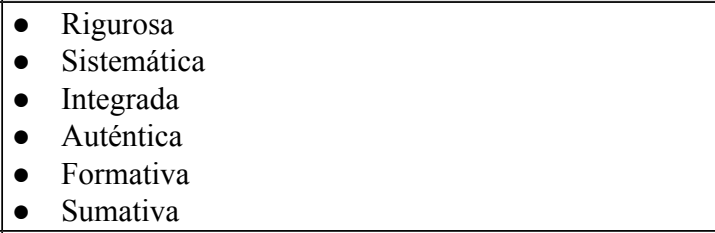 \\
\hline $\begin{array}{l}\text { ¿Al servicio de quién } \\
\text { está la evaluación de los } \\
\text { aprendizajes? }\end{array}$ & 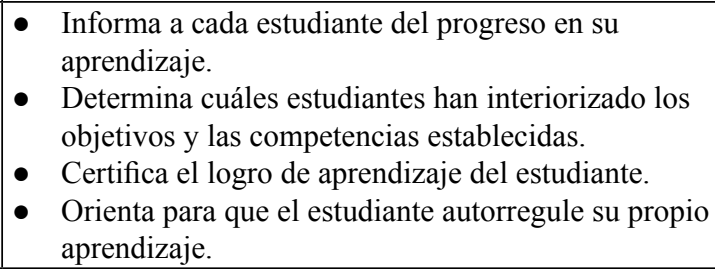 \\
\hline $\begin{array}{l}\text { ¿Cuál es la planificación } \\
\text { de la evaluación de los } \\
\text { aprendizajes? }\end{array}$ & $\begin{array}{l}\text { Las propuestas o los tipos de evaluación de los } \\
\text { aprendizajes deben ser definidos desde el diseño de } \\
\text { la asignatura e incorporados en las Orientaciones } \\
\text { Académicas de la asignatura. } \\
\text { - Los tipos de evaluaciones deberán ser planificados } \\
\text { e informados a cada estudiante para cada período } \\
\text { académico. }\end{array}$ \\
\hline
\end{tabular}

Nota: Adaptado del RGE (UNED, 2012a), análisis hecho en 2017.

En este reglamento, se determinan características de la evaluación de los aprendizajes referidas a su utilidad y uso. En este sentido, la evaluación informa del progreso de aprendizaje a cada estudiante e identifica quiénes han interiorizado los objetivos de aprendizaje establecidos. Otro uso que se da a la evaluación es para certificar el logro de los aprendizajes individualmente, lo cual es comprendido como consecuencia del proceso de enseñanza y aprendizaje.

\section{Plan de Desarrollo Académico 2012-2017 de la UNED}

Este Plan es una propuesta de trabajo planteada por la Vicerrectoría Académica como instrumento para la mejora continua, con el propósito de fortalecer la modalidad a distancia y cumplir con la misión institucional de darle prioridad a las poblaciones vulnerables, desde la cobertura, la calidad y la pertinencia académica (UNED, 2012b). 
Las dimensiones trazadas en el plan mencionado establecen los temas prioritarios para el quinquenio de la UNED. En este sentido, se identifican como dimensiones la mediación pedagógica, la evaluación para aprender, la territorialidad académica y las tecnologías digitales para el aprendizaje, las cuales deben ser articuladas entre sí y fortalecidas desde el quehacer cotidiano de la academia a partir del trabajo colaborativo y los principios éticos (UNED, 2012b).

La evaluación para aprender pretende que las prácticas evaluativas contribuyan en el aprendizaje del estudiantado, lo que promueve la autonomía y la autorregulación a través de la evaluación formativa y formadora. Igualmente, plantea la necesidad de ejecutar la evaluación de los aprendizajes desde sus tres tipos: diagnóstica, formativa y sumativa, utilizando estrategias alternativas de evaluación.

En este sentido, el Plan de Desarrollo Académico define evaluar para aprender como "un proceso continuo, formativo y formador inmerso en el aprendizaje. No son momentos separados ni paralelos, sino articulados. Lo relevante es quien aprende y la meta es el aprendizaje del estudiante" (UNED, 2012b, p. 43).

En el Plan de Desarrollo Académico, queda explícito el tema ético, el cual es un atributo esencial en cualquier nivel o campo de la evaluación educativa. Además, define la evaluación de los aprendizajes desde el enfoque de evaluar para aprender, entendida entonces como "un proceso continuo, formativo y formador inmerso en el aprendizaje. No son momentos separados ni paralelos, sino articulados. Lo relevante es quien aprende y la meta es el aprendizaje del estudiante" (UNED, 2012b, p. 43), (ver Tabla 3). 
Tabla 3.

Caracterización de la evaluación de los aprendizajes según el Plan de Desarrollo Académico (UNED 2012b)

\begin{tabular}{|c|c|}
\hline Consideración & Característica \\
\hline $\begin{array}{l}\text { ¿Cómo debe ser la } \\
\text { evaluación de los } \\
\text { aprendizajes? }\end{array}$ & $\begin{array}{l}\text { - Proceso continuo inmerso en el aprendizaje } \\
\text { - Formativa } \\
\text { - Formadora } \\
\text { - Sumativa }\end{array}$ \\
\hline $\begin{array}{l}\text { ¿Al servicio de quién } \\
\text { está la evaluación de los } \\
\text { aprendizajes? }\end{array}$ & $\begin{array}{l}\text { - Lo relevante es quien aprende } \\
\text { - La meta es el aprendizaje del estudiante }\end{array}$ \\
\hline $\begin{array}{l}\text { ¿Cuál es la planificación } \\
\text { de la evaluación de los } \\
\text { aprendizajes? }\end{array}$ & $\begin{array}{l}\text { - Las formas evaluativas deben construirse con } \\
\text { principios éticos } \\
\text { - Las experiencias evaluativas deben realizarse con } \\
\text { principios técnicos confiables }\end{array}$ \\
\hline $\begin{array}{l}\text { ¿Cuál es la función de } \\
\text { la evaluación? }\end{array}$ & $\begin{array}{l}\text { - Otorgar información de retorno } \\
\text { - Brindar datos relevantes } \\
\text { - Certificar con confiabilidad } \\
\text { - Evaluar para aprender }\end{array}$ \\
\hline $\begin{array}{l}\text { ¿Qué tipo de evaluación } \\
\text { según sus agentes? }\end{array}$ & - Promueve la coevaluación \\
\hline
\end{tabular}

Nota: Adaptado de UNED (2012b), análisis hecho en 2017.

\section{Cómo diseñar y ofertar cursos en línea: consideraciones generales (UNED, 2011) \\ El documento denominado Cómo diseñar y ofertar cursos en lí-} nea: consideraciones generales (UNED, 2011) surge como respuesta de capacitación a docentes en el tema de asignaturas o cursos ofertados a través del entorno virtual de aprendizaje. Dicho documento busca describir los fundamentos didácticos necesarios para que las asignaturas en línea trasciendan lo tecnológico y se enfoquen en el aprendizaje del estudiantado. A la vez, este documento conceptualiza la evaluación de los aprendizajes propuesta por Jorba y Casellas (1997) según se citan en la UNED, (2011), se destaca que debe entenderse como "un proceso donde, al final, se lleva a cabo un juicio de desempeño del estudiante, a partir de los diversos momentos de medición que se realizan en el curso" (p. 58). En este sentido, se puede afirmar que la evaluación de los aprendizajes apoyada en entornos virtuales implica desarrollar e incorporar diversas estrategias e instrumentos que servirán como evidencia para la valoración que se haga del logro o desempeño del estudiantado en relación con su aprendizaje. 
La evaluación planteada en el documento Cómo diseñar y ofertar cursos en línea (UNED, 2011) hace referencia a formas evaluativas más alternativas, como la autoevaluación, la coevaluación y la evaluación continua; a pesar de estar enfocada en momentos de medición y final, dado que el documento presenta la definición de Jorba y Castilla (1997), quienes la establecen como "un proceso donde, al final, se lleva a cabo un juicio de desempeño del estudiante, a partir de los diversos momentos de medición que se realizan en el curso" según se cita en la UNED (2011, p. 50), (ver Tabla 4).

\section{Tabla 4.}

Caracterización de la evaluación de los aprendizajes según el instructivo titulado Cómo diseñar y ofertar cursos en línea (UNED, 2011)

\begin{tabular}{|c|c|}
\hline Consideración & Característica \\
\hline $\begin{array}{l}\text { ¿Cómo debe ser la evaluación de } \\
\text { los aprendizajes? }\end{array}$ & $\begin{array}{ll}\text { - } & \text { Continua } \\
\text { - } & \text { Diagnóstica } \\
\text { - } & \text { Formativa } \\
\text { - } & \text { Sumativa }\end{array}$ \\
\hline $\begin{array}{l}\text { ¿Al servicio de quién está la } \\
\text { evaluación de los aprendizajes? }\end{array}$ & $\begin{array}{l}\text { - Permite que el estudiante monitoree su } \\
\text { nivel de avance. }\end{array}$ \\
\hline $\begin{array}{l}\text { ¿Cuál es la planificación de la } \\
\text { evaluación de la evaluación de los } \\
\text { aprendizajes? }\end{array}$ & $\begin{array}{l}\text { - Los criterios de evaluación deben darse con } \\
\text { anterioridad. }\end{array}$ \\
\hline $\begin{array}{l}\text { ¿Cuál es la función de la } \\
\text { evaluación? }\end{array}$ & $\begin{array}{l}\text { - Realimentación del proceso de enseñanza y } \\
\text { aprendizaje. }\end{array}$ \\
\hline $\begin{array}{l}\text { ¿Qué tipo de evaluación según sus } \\
\text { agentes? }\end{array}$ & $\begin{array}{ll}- & \text { Autoevaluación } \\
\text { - } & \text { Coevaluación }\end{array}$ \\
\hline
\end{tabular}

Nota: Adaptado de UNED (2011), análisis hecho en 2017.

Como se evidencia en el documento llamado Cómo diseñar $u$ ofertar cursos en línea (UNED, 2011), la evaluación de los aprendizajes debe ser continua y convertirse en una herramienta para que el estudiantado reconozca sus avances o limitaciones en el proceso educativo. Por lo tanto, esta debe impulsar la acción autorreguladora de los estudiantes, los cuales deben dedicar más atención a la forma en que identifican sus errores y los corrigen oportunamente. Así podrán llegar a ser previsores de sus limitaciones e implementar estrategias correctivas para superar sus propios resultados. 


\section{Conclusiones}

¿Cómo debe ser la evaluación de los aprendizajes en la UNED?

Se identificaron coincidencias entre el Modelo Pedagógico (UNED, 2004) y el Plan de Desarrollo Académico 2012-2017 (UNED, 2012b) al caracterizar la evaluación de los aprendizajes como una función reguladora, integrada, formativa y continua del proceso educativo. De los resultados obtenidos, llama la atención que no existe una única definición institucional de la evaluación de los aprendizajes que oriente el quehacer evaluativo en cada nivel y modalidad (tradicional a distancia o con apoyo tecnológico). Indistintamente de las diferencias en la conceptualización de la evaluación de los aprendizajes, la normativa revisada sí muestra características en común, tales como la autorregulación, la planificación, sus funciones formativas y sumativas, entre otras.

\section{en la UNED? \\ ¿Al servicio de quién está la evaluación de los aprendizajes}

Los resultados obtenidos a partir de la normativa institucional de la UNED permiten afirmar que la evaluación de los aprendizajes en una institución con modalidad educativa a distancia debe caracterizarse por estar al servicio del estudiante, así como por promover la autoevaluación y la coevaluación, y también por cumplir con la rigurosidad ética y técnica. Sus funciones principales son la realimentación del proceso y la certificación del logro del aprendizaje. Además, debe ser diagnóstica, formativa, formadora y sumativa, también auténtica y continua.

Los docentes y los estudiantes necesitan trascender la caracterización tradicional de la evaluación, que enfatiza la certificación de aprendizaje a partir de un momento único y sumativo, y dar más importancia a los usos, como la realimentación oportuna y correctiva.

Por consiguiente, es importante destacar que la normativa institucional de la UNED se caracteriza por presentar la evaluación de los aprendizajes como un proceso inherente, necesario y continuo en la formación académica del estudiantado universitario. Por ende, se le confiere un propósito orientador, preventivo y correctivo, tanto para el quehacer docente, como para el accionar del estudiante. 


\section{¿Qué aspectos implica la planificación de la evaluación de los aprendizajes?}

Puede concluirse que caracterizar la evaluación de los aprendizajes en la normativa institucional no es suficiente, ya que se requiere la operacionalización de dichas atribuciones para orientar las prácticas evaluativas de cada asignatura. Entre ellas se encuentran: tipos, funciones, agentes y momentos de la evaluación. También es preciso generar mecanismos de control que permitan verificar su cumplimiento.

En un modelo de educación a distancia, la planificación de la evaluación de los aprendizajes en entornos virtuales requiere concordancia con las características y los atributos que se expresan en la normativa institucional. Cuando se está en la etapa de planificación, se requiere considerar los atributos que la institución le ha conferido a la evaluación de los aprendizajes apoyada en entornos virtuales. Esto con el objetivo de operacionalizar prácticas alternativas de evaluación y, a la vez, generar los controles para verificar su aplicación.

\section{¿Cuál es la función de la evaluación?}

Se identificó que la función de la evaluación es orientar la planificación, autónomamente, de las acciones que conducen al aprendizaje, más allá de ser la evaluación una medición o comprobación de resultados para brindar realimentación en el proceso.

Una caracterización adecuada de la evaluación de los aprendizajes permite al estudiantado identificar sus habilidades de planificación y gestión del aprendizaje autónomo, con el propósito de aprovechar los materiales, los recursos y las estrategias evaluativas que le permitirán moldear y formar su aprendizaje; de acuerdo con los objetivos previamente definidos por el docente en el currículo de cada asignatura.

Las prácticas evaluativas que promuevan la autonomía, la criticidad y la producción académica tendrán como consecuencias estudiantes con niveles considerables de producción y generación de conocimiento autorregulado.

\section{¿Cuál es el tipo de evaluación según sus agentes?}

Uno de los aspectos de coincidencia en los documentos fue el compromiso de asumir como estrategia la autoevaluación y la coevaluación de los estudiantes. De tal manera que la evaluación de los aprendizajes en entornos virtuales, cuando es asumida como un 
proceso continuo y regulador, promueve la autonomía estudiantil. A la vez, desarrolla habilidades y actitudes autoevaluadoras del propio proceso de aprendizaje.

\section{Referencias}

Asamblea de la República de Costa Rica. (12 de marzo de 1977). Ley n. ${ }^{\circ}$ 6044: Creación de la Universidad Estatal a Distancia. [Ley 6044]. San José, Costa Rica: La Gaceta, (50).

Barberà, E., Romiszowski, A., Sangrà, A. y Simonson, M. (2014). Educación abierta y a distancia. Barcelona: Editorial UOC.

Calderón, Y. (2017). Valoración de las implicaciones éticas, sociales y didácticas de la evaluación de los aprendizajes apoyada en entornos virtuales (Tesis de Maestría Académica en Evaluación Educativa). Universidad de Costa Rica, San José, Costa Rica:

Cardona, J. (1994). Metodología innovadora de evaluación de centros educativos. Madrid: Ed. Sanz y Torres.

Casanova, M. (1995). Manual de evaluación educativa. Madrid: La Muralla.

Castillo, S. (2002). Compromisos de la Evaluación Educativa. Madrid: Pearson Education.

Castillo S. y Cabrerizo D. (2007). Evaluación educativa y promoción escolar. Madrid: Pearson Education.

Castillo S. y Cabrerizo D. (2009). Evaluación Educativa de aprendizaje y competencias. Madrid: UNED y Pearson Education.

De Ketele, J. M. y Roegiers, X. (1995). Metodología para la recogida de información. Madrid: La Muralla.

Esquivel, J. M. (2009). Evaluación de los aprendizajes en el aula: una conceptualización renovada. En E. Martín y F. Martínez (Coords.). Avances y desafios en la evaluación educativa (pp. 127-143). Madrid: Organización de Estados Centroamericanos.

Flores, C. (2010). Evaluación de los aprendizajes en la universidad. Revista Blanco y Negro, 1, 1-6. Recuperado de http://revistas.pucp. edu.pe/index.php/enblancoynegro/article/view/2189

Jorba, J. y Casellas, E. (1997). La regulación y la autorregulación de los aprendizajes. Barcelona: Síntesis.

Juca, F.X. (2016). La educación a distancia, una necesidad para la formación de los profesionales. Revista Universidad y Sociedad, 8(1), 106-111. Recuperado de http://scielo.sld.cu/scielo. php?script=sci_arttext\&pid=S2218-36202016000100016 
López, V. M. (Coord.). (2009). Evaluación Formativa y Compartida en Educación Superior. Propuestas, técnicas, instrumentos y experiencias. Madrid: Narcea.

Medina, A., Cardona, J., Castillo, S. y Domínguez, C. (1998). Evaluación de los procesos y los resultados del aprendizaje de los estudiantes. Madrid: UNED.

Morgan, Ch. y O'Relly, M. (2002). Assessing Open and Distance Learners. Londres: Kogan Page.

Perazzi, M. y Celma, S. (2017). La evaluación de los aprendizajes en aulas universitarias: una investigación sobre las prácticas. Praxis educativa, 21(3), 23-31. DOI: 10.19137/ praxiseducatva-2017-210303

Pimienta, J. (2008). Evaluación de los Aprendizajes. Un enfoque basado en competencias. México: Ed. Pearson y Prentice Hall.

Quesada, R. (2006). Evaluación del aprendizaje de la educación a distancia "en línea". Recuperado de https://revistas.um.es/red/ article/view/24291

Reina, J. (1993). Proyecto Curricular de Educación Primaria. Madrid: Escuela Española.

Rotger Amengual, B. (1992). Evaluación Formativa. Madrid: Ed. Cincel. Sanmartí, N., Jorba, J. y Ibáñez, V. (2002). Aprender a regular y autorregularse. En Pozo, J. I. y Monereo, C. (Coords.), El aprendizaje Estratégico. Enseñar a aprender desde el currículo (pp. 301-322). Madrid: Santillana.

Universidad Estatal a Distancia. (2004). Modelo Pedagógico. Recuperado de https://www.uned.ac.cr/conuniversitario/politicas/ politicas-academicas/modelo-pedagógico

Universidad Estatal a Distancia. (2011). Cómo diseñar y ofertar cursos en línea. Recuperado de https://www.uned.ac.cr/academica/ pace/recursos/modulos-para-diseno-de-cursos-asignaturas

Universidad Estatal a Distancia. (2012a). Reglamento General Estudiantil. Recuperado de http://www.uned.ac.cr/academica/images/cidreb/reglamento/estudiantil/general_estudiantil.pdf

Universidad Estatal a Distancia. (2012b). Plan de Desarrollo Académico. Recuperado de http://www.uned.ac.cr/academica/plan_academico/insumos/PlanDesAcad_UNED_final.pdf 